\title{
Circadian Clock Regulation through Pre-mRNA Splicing: A Review
}

\author{
Saipayan Ghosh ${ }^{1}$, Mainak Barman ${ }^{2}$, Sikha Snehal ${ }^{1}$ and Pratik Kumar ${ }^{1}$ \\ ${ }^{1}$ Department of Agricultural Biotechnology and Molecular Biology, \\ DRPCAU, Pusa, Bihar, India \\ ${ }^{2}$ Department of Plant Breeding and Genetics, DRPCAU, Pusa, Bihar, India \\ *Corresponding author
}

\section{A B S T R A C T}

\section{Keywords \\ Alternative splicing, Circadian clock, pre-mRNA splicing, Circadian clock regulation, Spliceosome \\ Article Info \\ Accepted: \\ 04 March 2020 \\ Available Online: \\ 10 April 2020}

Alternative splicing has a crucial role in the regulation of gene functions and enhancement of the proteome diversity in plants. In plants especially Arabidopsis, introns are present in most of the genes. A significant number of the intron-split genes concerned with the regulation of different biological events counting the circadian clock are alternatively spliced. In this current review, our main aim is to study the participation of alternative splicing in the circadian clock regulation.

\section{Introduction}

The earth undergoes rotation while revolving around the sun. This is responsible for alteration is of light and dark periods leading to variation in temperature. The variation of day length in different locations has been reported due to the changing of the sun's positions to the earth's axis. The mechanism that is responsible for the prediction of such light and dark alterations is referred to as the biological clock, or in a more scientific term, the circadian clock after the Latin, "circa diem", meaning "around or about a day". The "clock" works in a rhythm of about 24 hours indicating the time of day, which is almost indicative of the length of the day. On the other hand, seasonal variations can also be estimated by calculating day length, which is very highly significant both at the equator and higher latitudes. Erwin Bunning, a German plant physiologist researched circadian rhythms and concluded that these rhythms have an adaptive value (Bünning, 1964).

\section{History of the plant clock}

In plants, molecular research picked up the pace when Steve Kay and Andrew Millar introduced the firefly Luciferase (LUC) 
reporter gene, fused with the photosynthesis gene Chlorophyll $A / B$ Binding Protein 2 (CAB2) (Millar and Kay, 1991) into the model plant Arabidopsis thaliana. CAB2 expression has been reported to be regulated both by the circadian clock as well as by the phytochrome (phy) photoreceptors (Millar and Kay, 1991; Anderson et al., 1994). Photosynthesis is an important physiological process responsible for plant growth connecting a large number of plant circadian rhythms (Millar, 1999).

The possible task of the clock in the lightharvesting process is to anticipate the light period to get the plant ready for photosynthesis. Hence, energy uptake efficiency throughout the day has been enhanced by having the photosynthetic machinery being activated from the first light. The circadian clock has a significant role in regulating the different metabolic and developmental events to the most favorable time of the day, thereby enhancing the probability of growth and chance of survival of microorganisms (Blasing et al., 2005; Dodd et al., 2005).

\section{Genetics of plant clock}

Most of the attempts to reveal different mechanisms of biological clocks in different species have been conducted on mutants having phenotypes with a malfunctioning clock. Mutants developed through different forward and reverse genetic approaches led to the discovery of genes coding for proteins having normal circadian rhythms. In forward genetics, the selection is based upon a phenotype. In clock research, a changed phase of clock regulated gene expression is a breeding objective. In reverse genetics, selection of traits is done from genotype to phenotype and accordingly effect of gene manipulation is studied. Through mutational analyses, complex systems are studied by alterations of a single component while keeping all other parts constant (Dunlap, 1993). On the other hand, the resulting phenotype is formed due to the interaction of many factors. Such genetic studies are intended towards providing insight into the rhythmic biochemistry of the biological clock (Dunlap, 1993).

Screening of the mutants having relevant phenotypes is comparatively easier. On the other hand, it is more challenging to define the function of the mutated gene within the clock system. Based upon the locus of the induced mutation within a gene, there can be considerable variation in phenotype, e.g. toc 1 1 mutants have a phenotype that is dependent to light, but not the toc1-2 mutants (Millar et al., 1995; Somers et al., 1998; Strayer et al., 2000; Más et al., 2003). The most significant example of such type of mutant is a gene knock-out, in which the mutation or insertion is frequently located within an exon and hence drastically affects transcription/ translation.

However, non-functional mutants may also be obtained, in which transcription of the mutated gene might take place, its wild-type function is not possible. For example, a synthesized protein may not bind with its substrate due to a conformational change in the protein or lack of phosphorylation. A third type is a mutant with gain-in-function, in which the mutated gene/protein gains a new function, for instance, over-expression mutants, may constantly promote expression of another protein.

It is not easy to predict the sorts of mutagenesis taken up by clock mutant from their clock phenotypes. A knock-out disrupts the rhythm in no way, there is the shortening of increasing phase and/or period. To disrupt the clock completely, several genes have to be knocked-out or they need to over-express the 
right component (Wang and Tobin, 1998; Ding et al., 2007). An additional factor to consider is the timing; to disrupt the interaction between two proteins at a certain time of day may lead to an out-of-phase clock, while at protein levels remain there no change. Bearing all these variables in mind, it is highly challenging to firmly establish mutation types and their effects, for defining a new piece of the clock machinery.

\section{Pre-mRNA splicing machinery: the spliceosome}

mRNA is produced as a precursor mRNA (pre-mRNA) during the process of transcription in the nucleus (Wahl et al., 2009). There, it undergoes a series of processing steps before being transported to the cytoplasm where it serves as a template for protein biosynthesis and is degraded eventually (Wahl et al., 2009).

One of the processing steps is the elimination of introns from the intron-containing premRNAs, which is termed pre-mRNA splicing. In eukaryotes, pre-mRNA splicing is one of the fundamental processes in constitutive and regulated gene expression, as most genes typically contain multiple introns (Moore and Proudfoot, 2009).

The removal of introns from the pre-mRNA is involved in sequential phosphodiester transfer reactions which are catalyzed by the spliceosome, a large ribonucleoprotein (RNP) complex (Wang and Burge, 2008). The spliceosome is one of the most complex machines in the cell (Zhou et al., 2002; Jurica and Moore, 2003), consisting of five uridinerich (U-rich) small nuclear RNAs (snRNAs $\mathrm{U} 1, \mathrm{U} 2, \mathrm{U} 4, \mathrm{U} 5$, and U6), five small nuclear RNPs (snRNPs), and a multitude of nonsnRNP splicing factors, such as serine/ arginine-rich (SR) proteins (Deckert et al., 2006). The spliceosome assembly which begins anew at each intron guided by consensus sequences located in the premRNA is a highly ordered and dynamic reaction (Wahl et al., 2009). During the course of splicing, the sequences of exon and intron have to be recognized effectively and appropriate $5^{\prime}$ and $3^{\prime}$ splice sites (5'-SS and $3^{\prime}$ SS) have to be chosen before the catalytic step (Wang and Burge, 2008).

Three conserved elements in introns of the pre-mRNAs that are cis-acting comprise of the 5'-SS with a conserved GU dinucleotide, the 3 '-SS with a conserved AG dinucleotide, in addition to the branch point sequence (BPS) with a conserved UACUAAC sequence in yeast, but little conserved BPS in other higher eukaryotes located about 18-40 nt upstream of the 3'-SS (Wang and Burge, 2008). The splicing complexes help in recognizing these elements and take part in regulating the splicing reactions.

The assembly procedure of the spliceosome is exceedingly dynamic by the formation of several intermediate complexes, referred to as E, A, B, and B* (Deckert et al., 2006; Will and Luhrmann, 2011). The interaction of U1 snRNP takes place with the conserved 5'-SS, resulting in the complex $\mathrm{E}$ or early presplicing complex.

At this point, the U2 snRNP is related loosely to the pre-mRNA (Das et al., 2000; Deckert et al., 2006). Afterward, the U2 snRNP carries out stable interaction with the BPS of the premRNA, leading to the construction of the complex A or pre-spliceosome dependent on the hydrolysis of ATP (Deckert et al., 2006).

As a final point, the pre-formed U4/U6/U5 tri-snRNP particle adheres to the A complex and develops the spliceosomal B complex. This includes a full set of U snRNAs in a precatalytic state (Deckert et al., 2006; Wahl et al., 2009). Following a series of 
conformational as well as compositional alterations, counting the release of the $\mathrm{U} 1$ and U4 snRNPs, the catalytic actions of the spliceosomal B complex are activated and forms the $\mathrm{B}^{*}$ complex, the so-called activated spliceosome, to execute the sequential transport of phosphodiester (Smith et al., 2008; Wahl et al., 2009).

A two-step mechanism catalyzes the splicing (Deckert et al., 2006; Smith et al., 2008). In the first step, the $5^{\prime}-\mathrm{SS}$ is cleaved, and the $5^{\prime}$ end of the intron is linked covalently to the BPS, outlining a lariat structure. During the next step of splicing, the 3'-SS is cleaved, making the intron release and ligation of the $5^{\prime}$ and $3^{\prime}$ ends of the exons takes place for the purpose of forming the mRNA (Deckert et al., 2006).

Upon disassembly of the spliceosome, both the pre-mRNA splicing products and the components of the spliceosome are released finally, and the individual subunits of the spliceosome join in subsequent rounds of splicing. The composition of the spliceosomes might be comparable to that of the animal spliceosome since many components of the spliceosomes in animals have their presence in plants, signifying that the basal mechanisms in plants are similar to that of other organisms (Lorkovic et al., 2000; Reddy, 2004).

The 5' and 3'-SS in all the introns of Arabidopsis and rice analyzed are very similar to those of human beings, but the noncanonical splice sites, come about in only $0.7 \%$ of all splice sites, slightly lesser than the percentage found in the animals (Reddy, 2007). Moreover, the branch point sequence (CURAY) is not obvious in plants because of the difference in the position of the branch point in diverse introns, signifying that the mechanisms concerned in splice site recognition perhaps vary in these organisms (Reddy, 2007).
Significance of alternative splicing (AS) in plants

It is an indispensable post-transcriptional regulatory mechanism that can regulate gene expression, as well as extend transcriptome plasticity and proteome diversity in the eukaryotes (Stamm et al., 2005). Manifold transcripts from a single gene can be generated by exon skipping, introns' retention, and/or selection of an alternative 5' or 3'-SS (Reddy, 2007).

The events of intron retention often generate mRNAs with premature termination codons (PTCs) (Maquat, 2004; Reddy, 2007). The mRNAs with PTCs can be translated into truncated proteins, lacking a few active domains that are present in the full-length protein. These truncated proteins can contribute to the control of the quantity of functional protein that is produced, which has been demonstrated for many genes in plants that are related to development as well as disease resistance (Dinesh-Kumar and Baker, 2000; Zhang and Gassmann, 2003; Seo et al., 2012). Several variants of splicing are fixed as functional AS events in order to regulate gene expression or define the functions of certain proteins in plants, though the functions of only a limited AS events have been revealed in plants so far (Wang and Brendel, 2006).

AS is a vital mechanism for regulating the function of genes and boosting the coding potential of a genome in plants (Lorkovic et al., 2000; Reddy, 2007). It plays essential tasks in regulating the development of plants and tolerance to biotic as well as abiotic stresses. On the contrary, AS is regulated by development and environmental stresses also (Brett et al., 2002; Palusa et al., 2007; Filichkin et al., 2010). AS of Flowering Time Control Locus A (FCA) results in four transcripts $(\alpha, \beta, \gamma$, and $\delta)$, which are essential in the autoregulation of its expression and the 
control of the floral transition (Macknight et al., 1997). Prejudicing the function of SR45, a protein that is specific to plant, consequences in a splicing defect, afterward floral transition, in addition to aberrant leaf morphology phenotypes in Arabidopsis (Zhang and Mount, 2009; Tanabe et al., 2009). AS of most SR genes is altered by abiotic stresses strongly. Alternatively spliced forms of SR1 are regulated by high temperature (Lazar and Goodman, 2000).

\section{Regulatory functions of alternative splicing in the circadian clock}

Functions of $\mathrm{AS}$ in regulating the expression of the clock genes have been found out in recent times. More data in Arabidopsis discloses the importance of AS in the control of the clock (Wang and Ma, 2013). The clock-regulated Protein Arginine Methyl Transferase 5 (AtPRMT5) gene encodes a type II protein arginine methyltransferase that catalyzes the methylation of varied substrates (Deng et al., 2010). Mutations in atprmt5 trim down the methylation of the spliceosome components, such as AtSmD1 and AtLSm4, resulting in the splicing defects in genes engaged in many biological progressions (Sanchez et al., 2010; Deng et al., 2010). The circadian period is stretched by atprmt5 mutations (Hong et al., 2010). Imperfections in the alternative splicing of $P R R 7$ and $P R R 9$ in atprmt5-5 are responsible for the elongated period of the clock, first connecting AS to the clock (Sanchez et al., 2010).

Two additional splicing factors, Skiinteracting protein (SKIP) and Spliceosomal Timekeeper Locus1 (STIPL1), are engaged in the regulation of the circadian clock in Arabidopsis (Jones et al., 2012; Wang et al., 2012). STIPL1 is one another splicing factor linked with the regulation of the circadian clock, which encodes a homolog of TuftelinInteracting Protein11 (TFIP11) in the human- beings and Ntr1p in yeast is involved in disassembly of the spliceosome (Tannukit et al., 2009; Jones et al., 2012). These findings imply that the splicing factors, including AtSKIP and STIPL1, are necessitated for the accurate splicing of the genes related to circadian clock and for the normal functioning of the circadian clock. Thus, autoregulation of the transcription factors by generating competitive inhibitors through alternative splicing may be a common mechanism in their expression.

AS not regulates the functions of the circadian clock or the clock-related genes only, but also is under the control of the circadian clock (Staiger and Green, 2011). About 25-33\% of the protein-coding genes are regulated by the circadian clock at the entire-genome scale of Arabidopsis (Covington et al., 2008; Hazen et al., 2009). A number of other genes also demonstrate $>4 \mathrm{~h}$ phase differences between the oscillating introns and exons of the same transcriptional unit. A few genes have two splice forms- one is with but the other is without a cycling intron (Hazen et al., 2009).

Other than AS, microRNA (miRNA)mediated gene silencing is also an emerging regulatory mechanism to control the function of the circadian clock. MiRNAs are a plentiful group of endogenous, short (20-22 nt), single-stranded, non-coding RNAs that perform as post-transcriptional regulators of gene expression through sequence-specific cleavage or translational suppression of their target mRNAs in plants as well as animals (He and Hannon, 2004; Bartel, 2004). It has been recommended that each miRNA can control hundreds of target genes in humans, and $>60 \%$ of the protein-coding genes are the targets of miRNA (Friedman et al., 2009). The expression of miRNAs is regulated by the circadian clock (Cheng et al., 2007; Hazen et al., 2009) (Fig. 1 and 2). 


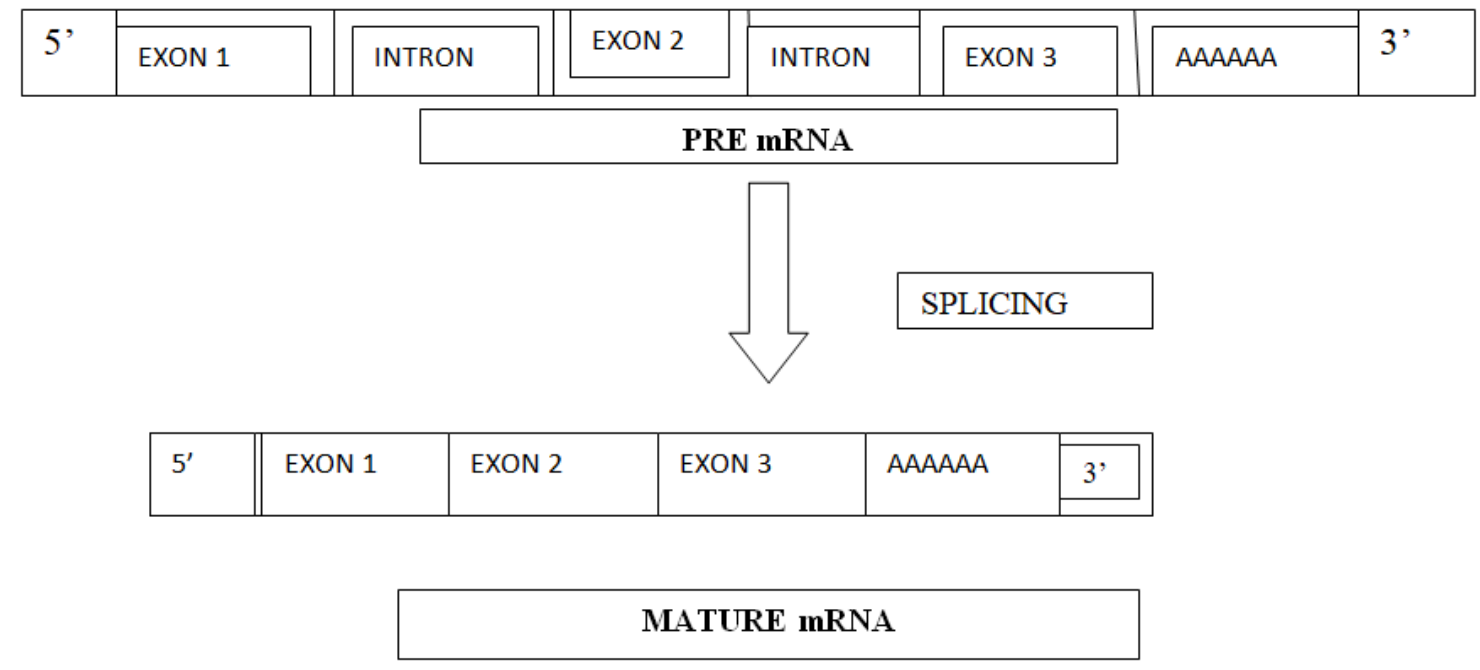

Figure.1 Mature-mRNA processing from Pre-mRNA

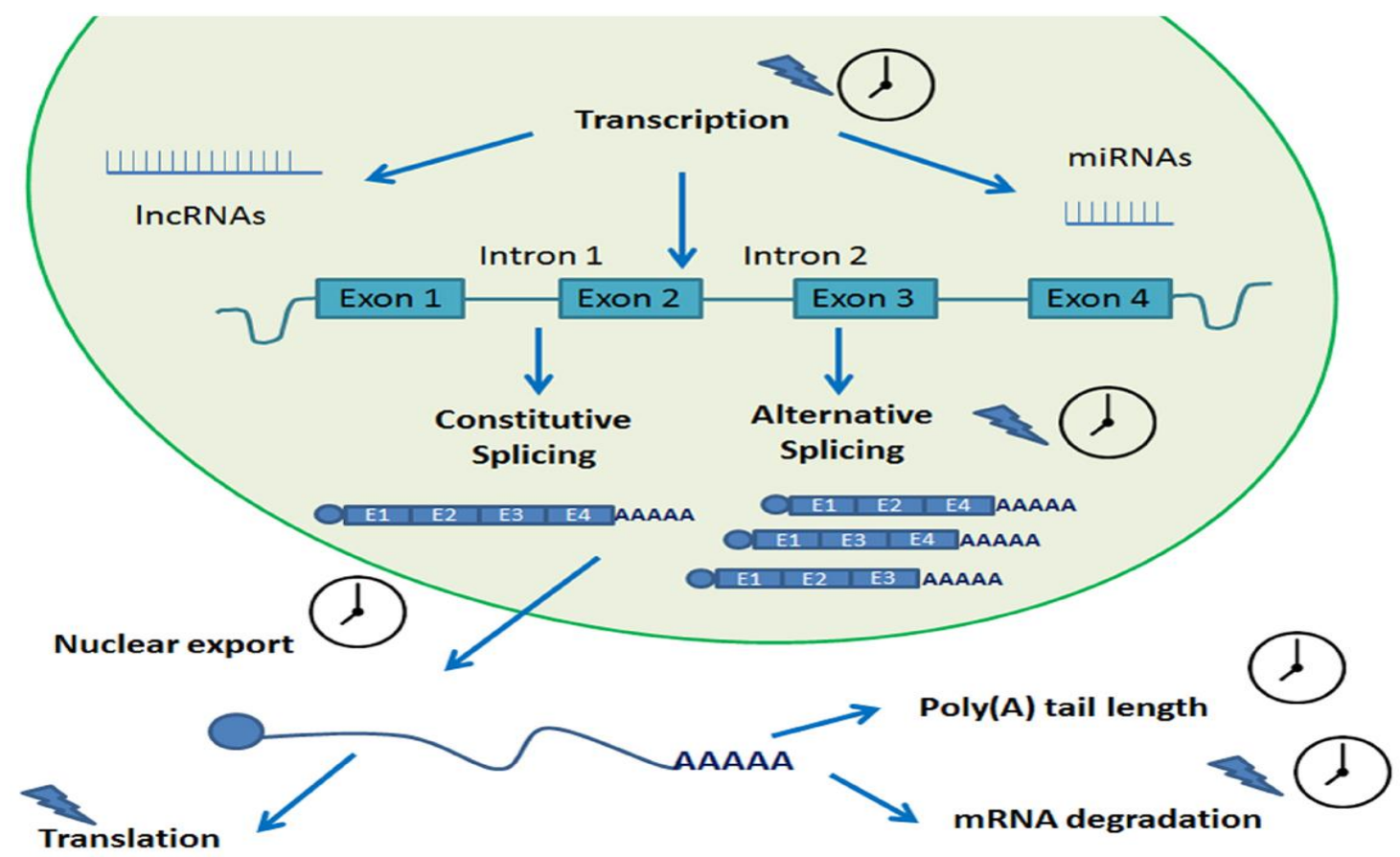

Figure.2 Post-transcriptional processes involved in circadian biology

(Romanowski and Yanovsky, 2015)

A number of miRNAs controlled by the circadian clock have been recognized in Arabidopsis by employing whole-genome TILLING arrays, including $m i R-160 b, m i R$ $167 d$, miR-158a, and miR-157a miRNAs in Arabidopsis (Hazen et al., 2009). In
Arabidopsis, photoperiodic flowering control is one of the circadian clock outputs that have been revealed to be under the regulation of miRNA (Schwab et al., 2005; Jung et al., 2007). The core components in the photoperiodic control flowering pathway, 
such as GI, COSTANS (CO), and Flowering Locus T (FT), show evidence of circadian rhythmicity (Hayama and Coupland, 2003).

To conclude we may say that the circadian clock is a vital mechanism in plants to synchronize the endogenous biological as well as biochemical procedures with the cues of the local day/night cycles. Even though AS is crucial for the normal functioning of the circadian clock, how AS regulates the circadian clock is a long way from obvious. Not only CCA1, but also TOC1, LHY, PRR3, PRR5, PRR7, PRR9, GI, ZTL, and other genes related to the circadian clock are subject to AS in Arabidopsis (Sanchez et al., 2010; Seo et al., 2012; James et al., 2012).

However, the molecular principles of their regulation of the circadian clock are still incomprehensible. An investigation in a detailed manner into how the AS of $L H Y$, PRR7, PRR9, TOC1, and GI genes plays their roles in regulating the circadian clock will elucidate the regulatory mechanisms of the circadian clock.

\section{References}

Anderson SL, Teakle GR, Martino-Catt SJ, et al., Circadian clock- and phytochromeregulated transcription is conferred by a 78 bp cis-acting domain of the Arabidopsis CAB2 promoter. Plant J. 1994; 6: 457-470.

Bartel DP. MicroRNAs: genomics, biogenesis, mechanism, and function. Cell. 2004; 116: 281-297.

Blasing OE, Gibon Y, Gunther M, et al., Sugars and circadian regulation make major contributions to the global regulation of diurnal gene expression in Arabidopsis. Plant Cell 2005; 17: 3257-3281.

Brett D, Pospisil H, Valcarcel J, et al., Alternative splicing and genome complexity. Nature Genetics. 2002; 30: 29-30.

Bünning E. Circadian Leaf Movements in Bean Plants - Earlier Reports. Science.1964: 146

Cheng HY, Papp JW, Varlamova O, et al.,
microRNA modulation of circadian-clock period and entrainment. Neuron. 2007; 54: 813-829.

Covington MF, Maloof JN, Straume M, et al., Global transcriptome analysis reveals circadian regulation of key pathways in plant growth and development. Genome Biology. 2008; 9: R130.

Das R, Zhou Z, Reed R. Functional association of U2 snRNP with the ATP-independent spliceosomal complex E. Molecular Cell. 2000; 5: 779-787.

Deckert J, Hartmuth K, Boehringer D, et al., Protein composition and electron microscopy structure of affinity-purified human spliceosomal B complexes isolated under physiological conditions. Molecular and Cellular Biology. 2006; 26: 55285543.

Deng X, Gu L, Liu C, et al., Arginine methylation mediated by the Arabidopsis homolog of PRMT5 is essential for proper pre-mRNA splicing. Proceedings of the National Academy of Sciences, USA. 2010; 107: 19114-19119.

Dinesh-Kumar SP, Baker BJ. Alternatively spliced $\mathrm{N}$ resistance gene transcripts: their possible role in tobacco mosaic virus resistance. Proceedings of the National Academy of Sciences, USA. 2000; 97: 1908-1913.

Ding Z, Doyle MR, Amasino RM, et al., A complex genetic interaction between Arabidopsis thaliana TOC1 and CCA1/LHY in driving the circadian clock and in output regulation. Genetics 2007; 176: $1501-1510$.

Dodd AN, Salathia N, Hall A, et al., Plant circadian clocks increase photosynthesis, growth, survival, and competitive advantage. Science. 2005; 309: 630-633.

Dunlap JC. Genetic analysis of circadian clocks. Annu Rev Physiol. 1993; 55: 683-728.

Filichkin SA, Priest HD, Givan SA, et al., Genome-wide mapping of alternative splicing in Arabidopsis thaliana. Genome Research. 2010; 20: 45-58.

Friedman RC, Farh KK, Burge CB, et al., Most mammalian mRNAs are conserved targets of microRNAs. Genome Research. 2009; 19: 92-105. 
Hayama R, Coupland G. Shedding light on the circadian clock and the photoperiodic control of flowering. Current Opinion in Plant Biology. 2003; 6: 13-19.

Hazen SP, Naef F, Quisel T, et al., Exploring the transcriptional landscape of plant circadian rhythms using genome tiling arrays. Genome Biology. 2009; 10: R17.

He L, Hannon GJ. MicroRNAs: small RNAs with a big role in gene regulation. Nature Reviews Genetics. 2004; 5(7): 522-31.

Hong S, Song HR, Lutz K, et al., Type II protein arginine methyltransferase 5 (PRMT5) is required for circadian period determination in Arabidopsis thaliana. Proceedings of the National Academy of Sciences, USA. 2010; 107: 21211-21216.

James $\mathrm{AB}$, Syed $\mathrm{NH}$, Bordage $\mathrm{S}$, et al., Alternative splicing mediates responses of the Arabidopsis circadian clock to temperature changes. The Plant Cell. 2012; 24: 961-981.

Jones MA, Williams BA, McNicol J, et al., Mutation of Arabidopsis spliceosomal timekeeper locus1 causes circadian clock defects. The Plant Cell. 2012; 24: 40664082.

Jung JH, Seo YH, Seo PJ, et al., The GIGANTEA-regulated microRNA172 mediates photoperiodic flowering independent of CONSTANS in Arabidopsis. The Plant Cell. 2007; 19: 2736-2748.

Jurica MS, Moore MJ. Pre-mRNA splicing: awash in a sea of proteins. Molecular Cell. 2003; 12: 5-14.

Lazar G, Goodman HM. The Arabidopsis splicing factor SR1 is regulated by alternative splicing. Plant Molecular Biology. 2000; 42: 571-581.

Lorkovic ZJ, Wieczorek Kirk DA, Lambermon $\mathrm{MH}$, et al., Pre-mRNA splicing in higher plants. Trends in Plant Science. 2000; 5: 160-167.

Macknight R, Bancroft I, Page T, et al., FCA, a gene controlling flowering time in Arabidopsis, encodes a protein containing RNA-binding domains. Cell. 1997; 89: 737-745.

Maquat LE. Nonsense-mediated mRNA decay: splicing, translation and mRNP dynamics.
Nature Reviews Molecular Cell Biology. 2004; 5: 89-99.

Más P, Kim WY, Somers DE, et al., Targeted degradation of TOC1 by ZTL modulates circadian function in Arabidopsis thaliana. Nature. 2003; 426: 567-570.

Millar AJ, Carre IA, Strayer CA, et al., Circadian clock mutants in Arabidopsis identified by luciferase imaging. Science. 1995; 267: 1161-1163

Millar AJ, Kay SA. Circadian Control of cab Gene Transcription and mRNA Accumulation in Arabidopsis. Plant Cell. 1991; 3: 541-550.

Millar AJ. Biological clocks in Arabidopsis thaliana. New Phytologist.1999; 141: 175197.

Moore MJ, Proudfoot NJ. Pre-mRNA processing reaches back to transcription and ahead to translation. Cell. 2009; 136: 688-700.

Palusa SG, Ali GS, Reddy AS. Alternative splicing of pre-mRNAs of Arabidopsis serine/arginine-rich proteins: regulation by hormones and stresses. The Plant Journa.l 2007; 49: 1091-1107.

Reddy AS. Alternative splicing of pre-messenger RNAs in plants in the genomic era. Annual Review of Plant Biology. 2007; 58: 267294.

Reddy AS. Plant serine/arginine-rich proteins and their role in premRNA splicing. Trends in Plant Science. 2004; 9: 541-547.

Romanowski A, Yanovsky MJ. Circadian rhythms and post-transcriptional regulation in higher plants. Front. plant sci. 2015;6: 437.

Sanchez SE, Petrillo E, Beckwith EJ, et al., A methyl transferase links the circadian clock to the regulation of alternative splicing. Nature. 2010; 468: 112-116.

Schwab R, Palatnik JF, Riester M, et al., Specific effects of microRNAs on the plant transcriptome. Developmental Cell. 2005; 8: 517-527.

Seo PJ, Park MJ, Lim MH, et al., A selfregulatory circuit of CIRCADIAN CLOCK-ASSOCIATED1 underlies the circadian clock regulation of temperature responses in Arabidopsis. The Plant Cell. 2012; 24: 2427-2442.

Seo PJ, Park MJ, Lim MH, et al., A selfregulatory circuit of CIRCADIAN CLOCK 
ASSOCIATED1 underlies the circadian clock regulation of temperature responses in Arabidopsis. The Plant Cell. 2012; 24: 2427-2442.

Smith DJ, Query CC, Konarska MM. 'Nought may endure but mutability': spliceosome dynamics and the regulation of splicing. Molecular Cell. 2008; 30: 657-666.

Somers DE, Devlin PF, Kay SA Phytochromes and cryptochromes in the entrainment of the Arabidopsis circadian clock. Science. 1998; 282: 1488-1490.

Staiger D, Green R. RNA-based regulation in the plant circadian clock. Trends in Plant Science. 2011; 16: 517-523.

Stamm S, Ben-Ari S, Rafalska I, et al., Function of alternative splicing. Gene. 2005; 344: 120.

Strayer C, Oyama T, Schultz TF, et al., Cloning of the Arabidopsis clock cone TOC1, an autoregulatory response regulator homolog. Science. 2000; 289: 768-771.

Tanabe N, Kimura A, Yoshimura K, et al., Plantspecific SR-related protein atSR45a interacts with spliceosomal proteins in plant nucleus. Plant Molecular Biology. 2009; 70: 241-252.

Tannukit S, Crabb TL, Hertel KJ, et al., Identification of a novel nuclear localization signal and speckle-targeting sequence of tuftelin-interacting protein 11, a splicing factor involved in spliceosome disassembly. Biochemical and Biophysical Research Communications. 2009; 390:1044-1050.

Wahl MC, Will CL, Luhrmann R. The spliceosome: design principles of a dynamic RNP machine. Cell. 2009; 136:
701-718.

Wang BB, Brendel V. Genomewide comparative analysis of alternative splicing in plants. Proceedings of the National Academy of Sciences, USA. 2006;103: 7175-7180.

Wang X, Ma L. Unraveling the circadian clock in Arabidopsis. Plant Signaling and Behavior. 2013; 8: e23014.

Wang X, Wu F, Xie Q, et al., SKIP is a component of the spliceosome linking alternative splicing and the circadian clock in Arabidopsis. The Plant Cell. 2012; 24: 3278-3295.

Wang Z, Burge CB. Splicing regulation: from a parts list of regulatory elements to an integrated splicing code. RNA. 2008; 14: 802-813.

Wang ZY, Tobin EM. Constitutive expression of the CIRCADIAN CLOCK ASSOCIATED 1 (CCA1) gene disrupts circadian rhythms and suppresses its own expression. Cell. 1998; 93: 1207-1217.

Will CL, Luhrmann R. Spliceosome structure and function. Cold Spring Harbor Perspectives in Biology. 2011;3: a003707.

Zhang XC, Gassmann W. RPS4-mediated disease resistance requires the combined presence of RPS4 transcripts with full-length and truncated open reading frames. The Plant Cell. 2003; 15: 2333-2342.

Zhang XN, Mount SM. Two alternatively spliced isoforms of the Arabidopsis SR45 protein have distinct roles during normal plant development. Plant Physiology. 2009; 150: 1450-1458.

Zhou Z, Licklider LJ, et al., Comprehensive proteomic analysis of the human spliceosome. Nature. 2002; 419: 182-185.

\section{How to cite this article:}

Saipayan Ghosh, Mainak Barman, Sikha Snehal and Pratik Kumar. 2020. Circadian Clock Regulation through Pre-Mrna Splicing: A Review. Int.J.Curr.Microbiol.App.Sci. 9(04): 371379. doi: https://doi.org/10.20546/ijcmas.2020.904.044 